\title{
SIMULATION OF CLOUD-AEROSOL LIDAR WITH ORTHOGONAL POLARIZATION (CALIOP) ATTENUATED BACKSCATTER PROFILES USING THE GLOBAL MODEL OF AEROSOL PROCESSES (GLOMAP)
}

\author{
Stuart Young $^{1}$, Martin Cope ${ }^{1}$, Sunhee Lee ${ }^{1}$, Kathryn Emmerson ${ }^{1}$, Matthew Woodhouse ${ }^{1}$,
} Nicolas Bellouin ${ }^{2}$

${ }^{\text {I} C S I R O ~ O c e a n s ~ \& ~ A t m o s p h e r e, ~ A s p e n d a l e, ~ V I C ~ 3195, ~ A u s t r a l i a, ~ * E m a i l: ~ s t u a r t . y o u n g @ c s i r o . a u ~}$

${ }^{2}$ Department of Meteorology, University of Reading, Reading, RG6 6BB, UK

\begin{abstract}
To permit the calculation of the radiative effects of atmospheric aerosols, we have linked our aerosol-chemical transport model (CTMGLOMAP) to a new radiation module (UKCARADAER). In order to help assess and improve the accuracy of the radiation code, in particular the height dependence of the predicted scattering, we have developed a module that simulates attenuated backscatter (ABS) profiles that would be measured by the satellite-borne Cloud-Aerosol Lidar with Orthogonal Polarization (CALIOP) if it were to sample an atmosphere with the same aerosol loading as predicted by the CTM. Initial results of our comparisons of the predicted ABS profiles with actual CALIOP data are encouraging but some differences are noted, particularly in marine boundary layers where the scattering is currently under-predicted and in dust layers where it is often over-predicted. The sources of these differences are being investigated.
\end{abstract}

\section{INTRODUCTION}

Atmospheric aerosol is well known to lead to perturbations in the radiation balance, via direct, and indirect (i.e. changes to cloud properties) effects. Accurate representation of the aerosol size distribution and characteristics such as solubility are essential if models are to simulate correctly the coupling between aerosol loading and radiative forcing.

Because the vertical distribution of aerosol layers in the atmosphere, in addition to their optical properties, can have a significant effect on their radiative impact, we consider it necessary to assess the accuracy of our model's predictions of those vertical profiles. As we apply our model over scales ranging from continental to global, we need to compare with vertically resolved measurements made on such scales. An ideal source of such data is the CALIOP lidar on board the CALIPSO satellite [1]. While it is common to compare model predictions with measurements of such quantities as aerosol optical depth or particulate extinction coefficient, it should be remembered that CALIOP is an elasticbackscatter lidar and does not measure either of these quantities directly. Rather it retrieves these quantities from its primary measurement, the attenuated backscatter coefficient, via a combination of analysis algorithms [1,2] and an optical model of the scattering properties of the aerosol (or cloud) particles. The optical model can only describe the average properties of an identified aerosol type. A misidentification of the aerosol type, or natural variation of the optical properties from the average values, will lead to errors, of various magnitudes, in the retrieved extinction and optical depths [3]. As a result, a difference between simulated and measured profiles of a certain optical quantity could be the consequence of an incorrect prediction of aerosol concentrations, location or type, or of optical properties on the side of the chemical transport model (CTM-GLOMAP), or of errors in the optical properties used to retrieve the profiles from the measurements. In some cases, this is effectively comparing one model with another, which is neither what is intended nor is it very informative. To overcome these problems, we choose instead to compare the fundamental lidar measured quantity, the attenuated backscatter profile, with simulated profiles derived from the radiation module (UKCA-RADAER) that is used by our CTM. Attenuated backscatter is the product of the sum of the molecular and particulate backscatter coefficients at any height and the molecular and particulate two-way transmittances between that height and the lidar. It is simply the calibrated backscattered power 
measured by the lidar corrected for the rangesquared decrease in signal with distance from the lidar. In this way all of the uncertainties are on one side of the comparison thus permitting unambiguous assessment of the model.

\section{METHODOLOGY}

\section{(a) The Models}

In our modeling system, continental-scale atmospheric transport is modeled using a coupled system that comprises (1) the CSIRO Conformal-Cubic Atmospheric Model [4,5] for simulating weather, (2) a population-based anthropogenic emission inventory with natural primary emission sources such as sea salt, windblown dust, plus biogenic and fire emissions, and (3) a chemical transport model (CTM,[6]) for simulating the atmospheric chemical transport and subsequent fate, via wet and dry deposition, of gaseous and particulate species. It has a comprehensive chemistry incorporating the Carbon Bond 5 mechanism [7], the Volatility Basis Set approach [8] for secondary organic aerosols, and the ISORROPIA-II model [9] for secondary inorganic aerosol modeling.

The CTM is coupled with the GLObal Model of Aerosol Processes (GLOMAP, [10]), which is a comprehensive, size-resolving global aerosol model. In this work CTM-GLOMAP employs 7 modes and 5 components. Its boundary and initial conditions for aerosol components and modes are obtained from an instance of GLOMAP running in a nudged global general circulation model. The CTM-GLOMAP domain for the Australia continent has a resolution of $0.5^{\circ}$.

The optical properties such as aerosol optical depth (AOD), and aerosol backscatter and extinction coefficients are calculated at each model grid by the RADAER module via a Mie look-up table for the appropriate sizes and refractive indices. The lidar signals are then simulated, as described below, at each of the 19 model levels, which extend from $0.02 \mathrm{~km}$ to $8 \mathrm{~km}$ above the surface, with a vertical resolution that varies from $0.02 \mathrm{~km}$ near the surface to $2 \mathrm{~km}$ at the top of the simulation. The comparison with the measurements is then currently done off line, by extracting simulated profiles from the model output file at each model grid point along the CALIPSO ground track of interest.

\section{(b) The Simulator}

In each model grid cell, the model system predicts the aerosol components and their size modes. For each of these aerosol components, the particulate backscatter, $\beta_{P}$, and extinction coefficients, $\sigma_{P}$, are then obtained at the CALIOP wavelengths (532 $\mathrm{nm}$ and $1064 \mathrm{~nm}$ ) via a pre-calculated Mie lookup table. In addition, the atmospheric temperature and pressure profiles, available from the modeled meteorology, can be used to calculate the molecular number density profile and, hence, the backscatter, $\beta_{\mathrm{M}}$, and extinction coefficient, $\sigma_{\mathrm{M}}$, profiles at the lidar wavelengths. The ozone number density is also available from the model allowing the ozone absorption profile, $\alpha_{\mathrm{O} 3}(\mathrm{z})$, to be calculated. (Ozone absorption is only significant at the shorter wavelength.) It is then a simple matter to calculate profiles of attenuated (total) backscatter:

$\beta_{T}^{\prime}(z)=\left[\beta_{M}(z)+\beta_{P}(z)\right] T_{M}^{2}\left(z_{S}, z\right) T_{P}^{2}\left(z_{S}, z\right)$,

where

$T_{M}^{2}\left(z_{S}, z\right)=\exp \left\{-2 \int_{Z_{S}}^{z}\left[\sigma_{M}(r)+\alpha_{O_{3}}(r)\right] d r\right\}$,

and

$$
T_{P}^{2}\left(z_{S}, z\right)=\exp \left\{-2 \int_{Z_{S}}^{z} \sigma_{P}(r) d r\right\}
$$

are, respectively, the molecular and particulate two-way transmittance profiles. The profile of attenuated scattering ratio, $R^{\prime}(\mathrm{z})$, is obtained from the attenuated total backscatter coefficient profile by dividing by the profile of attenuated molecular backscatter,

$$
\beta_{M}^{\prime}(z)=\beta_{M}(z) T_{M}^{2}\left(z_{s}, z\right),
$$

to give

$$
R^{\prime}(z)=\left[1+\beta_{P}(z) / \beta_{M}(z)\right] T_{P}^{2}\left(z_{S}, z\right) .
$$

To simulate the satellite-borne CALIOP profiles, the calculation is started at the top of the atmosphere (or the satellite altitude $z_{\mathrm{S}}$ ), and proceeds down towards the surface in order to account correctly for the increasing signal attenuation with range from the satellite (decreasing height). 


\section{(c) CALIOP Attenuated Backscatter Profiles}

The CALIOP data used in the comparisons here are the "Total_Attenuated_Backscatter_532" from the CALIPSO, level-1, version 3 files and the "Feature_Classification_Flags" in the corresponding, level-2, vertical feature mask (VFM) files.

Although the aim of this exercise is to compare measured and modeled profiles in an atmosphere containing air molecules and aerosol particles, in practice, the measured atmosphere often also contains clouds, which can complicate the comparison somewhat. To facilitate the comparisons, the measured data were filtered to exclude from the calculated average profiles all points in any single profile below the top of cloud layers that were identified in the corresponding VFM profiles. The filtered profiles were then averaged to produce a horizontal resolution comparable to that of the model in the domain of interest $\left(10^{\circ} \mathrm{S}-45^{\circ} \mathrm{S}, 110^{\circ} \mathrm{E}\right.$ to $\left.165^{\circ} \mathrm{E}\right)$. The vertical resolution of the measured data was, however, not degraded to that of the model but kept at $30 \mathrm{~m}$ over the complete height range.

\section{RESULTS}

In Figure 1 we present an example of our comparisons. The 532-nm attenuated total backscatter measured as CALIPSO passed over Australia on 7 October 2006 at approximately 1621 UTC is shown as a function of height and latitude in (a) with the simulation in (b). The aerosol subtype as identified by CALIPSO's algorithms is shown in (c). Notable features are a strongly scattering marine boundary layer extending to an altitude of $1 \mathrm{~km}$ to the North and $2 \mathrm{~km}$ to the South of the continent, and a deep moderately strongly scattering aerosol layer that extends to nearly $4 \mathrm{~km}$ altitude at latitudes of $20^{\circ}$ and $30^{\circ} \mathrm{S}$. Between these latitudes, the strength of the signal decreases markedly to the extent that it cannot be detected by the algorithms and does not appear in the VFM. CALIPSO's algorithms identify these aerosols as being predominantly "polluted dust" (a mixture of dust and smoke) with an indication that the weaker signal recorded between these latitudes is from pure dust.

The model generally correctly reproduces the location and heights of the marine layers, although the southern layer is slightly too low in places. The height of the continental layers is also fairly well reproduced as is the extension of the dust layer above the southern marine layer.

Although the horizontal and vertical locations of the various layers are correctly reproduced in the simulation shown, the magnitudes of the simulated signals are, in some places, rather different from what are observed.

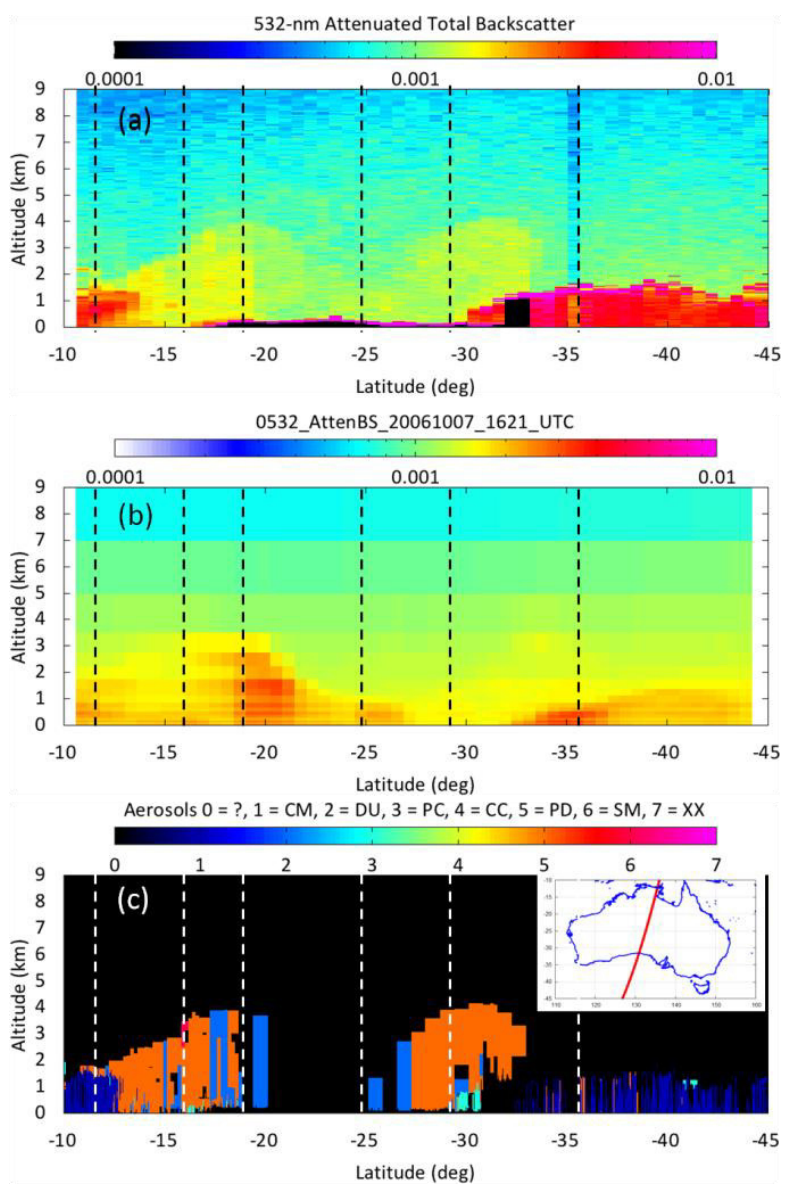

Figure 1. 532-nm Attenuated Total Backscatter (a) CALIOP, (b) Simulation. (c) Aerosol sub-type Vertical Feature Mask. $\mathrm{CM}=$ Clean Marine, $\mathrm{DU}=$ Dust, $\mathrm{PC}=$ Polluted Continental, $\mathrm{CC}=$ Clean Continental, $\mathrm{PD}=$ Polluted Dust, $\mathrm{SM}=$ Smoke, $\mathrm{XX}=$ not defined. Vertical, dashed lines indicate locations of profiles in Fig. 2. Inset shows CALIPSO ground track over Australia on 7 October 2006 at $\sim 1621$ UTC.

In order to study these differences in more detail, in Figure 2 we compare measured and modeled profiles of attenuated backscatter. The profiles correspond to the locations of the dashed vertical lines in Figure 1. Note that the magnitudes of the simulated profiles are larger than those of the 
measured profiles in the free troposphere. This is a result of the additional atmospheric attenuation that was measured between the lidar (at $705 \mathrm{~km}$ ) and the top of the simulation (at $\sim 9 \mathrm{~km}$ ) that has not been corrected for in these figures as it helps separate the profiles horizontally and, thereby, improve legibility. In the first profile, at $11.51^{\circ} \mathrm{S}$, this difference is increased by the attenuation caused by a layer of high cloud above $9 \mathrm{~km}$ and not shown here. The comparison of profiles shows the marked under-prediction of scattering in the marine layers in (a) and (f), reasonable agreement in the polluted dust in (b), but less so at (e), and a significant over-estimation of the signal from dust at (c) and (d). The extent to which these differences are attributable to incorrectly predicted number densities, hydration (for the marine layers) or optical properties (e.g. the use of Mie-scattering code for dust) is being investigated and will provide useful information for the development and improvement of our model and radiation code.

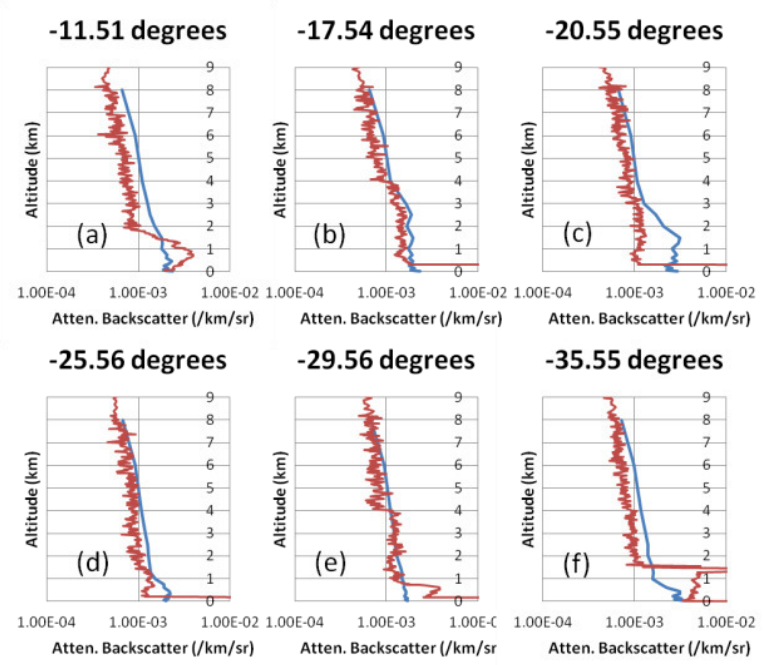

Figure 2. Profiles of 532-nm Attenuated Total Backscatter at the locations indicated by the dashed lines in Fig. 1. The red, noisy profiles are CALIOP data while the blue, smooth profiles are the simulations.

\section{ACKNOWLEDGEMENT}

The CALIPSO data were obtained from the NASA Langley Research Center Atmospheric Science Data Center.

\section{REFERENCES}

[1] Winker, D. M., et al., 2009: Overview of the CALIPSO Mission and CALIOP Data Processing
Algorithms, J. Atmos. Oceanic Technol., 26, 2310-2323.

[2] Young, S. A., and M. A. Vaughan, 2009: The Retrieval of Profiles of Particulate Extinction from Cloud-Aerosol Lidar Infrared Pathfinder Satellite Observations (CALIPSO) Data: Algorithm Description, J. Atmos. Oceanic Technol., 26, 1105-1119.

[3] Young, S. A. et al., 2013: The Retrieval of Profiles of Particulate Extinction from CloudAerosol Lidar Infrared Pathfinder Satellite Observations (CALIPSO) Data: Uncertainty and Error Sensitivity Analyses, J. Atmos. Oceanic Technol., 30, 395-428.

[4] McGregor, J. L., and M. R. Dix, 2001: The CSIRO conformal-cubic atmospheric GCM. In: UTAM Symposium on Advances in Mathematical Modelling of Atmosphere and Ocean Dynamics, P. F. Hodnett (Ed.), Kluwer, Dordrecht, 197-202.

[5] McGregor, J. L., and M. R. Dix, 2008: An updated description of the Conformal-Cubic Atmospheric Model, In: Hamilton K. and W. Ohfuchi (Eds.), High Resolution Simulation of the Atmosphere and Ocean Springer, 51-76.

[6] Cope, M. E., et al., 2009: Chemical Transport Model - Technical Description: Centre for Australian Weather and Climate Research, Technical Report 15, Melbourne, Australia.

[7] Sarwar, G., et al., 2008: Impact of an updated carbon bond mechanism on predictions from the CMAQ modeling system: Preliminary assessment, J. Appl. Meteorol. Clim., 47, 3-14.

[8] Donahue, N. M., et al., 2006: Coupled partitioning, dilution, and chemical aging of semivolatile organics, Environ. Sci. Technol., 40, 2635-2643.

[9] Fountoukis, C., and A. Nenes, 2007.: ISORROPIA II: a computationally efficient thermodynamic equilibrium model for $\mathrm{K}^{+}-\mathrm{Ca}^{2+}$ $\mathrm{Mg}^{2+}-\mathrm{NH}_{4}{ }^{+}-\mathrm{Na}^{+}-\mathrm{SO}_{4}{ }^{2-}-\mathrm{NO}_{3}{ }^{-}-\mathrm{Cl}^{-}-\mathrm{H}_{2} \mathrm{O}$ aerosols, Atmos. Chem. Phys, 7, 4639-4659.

[10] Mann, G. W., et al., 2010: Description and evaluation of GLOMAP-mode: a modal global aerosol microphysics model for the UKCA composition-climate model, Geosci. Model Dev., 3, 519-551. 